\title{
LA REHABILITACIÓN INTEGRADA DE LOS CENTROS HISTÓRICOS: EL RETO URBANÍSTICO DE FINALES DE LOS OCHENTA*
}

\author{
Antonio-José Campesino Fernández \\ Universidad de Extremadura
}

\begin{abstract}
RESUMEN
La quiebra del urbanismo positivista de los años sesenta y setenta precipita en los ochenta nuevos conceptos y prácticas de intervención en la ciudad. El urbanismo «recualificador» de la última generación en nuestros Centros Históricos se constituye en alternativa al ultraconservacionismo y a la renovación especulativa, evidenciando que la ciudad no es un instrumento de hacer dinero fácil y rápido, sino el marco de convivencia diaria sujeta a unas reglas claras de juego. No hay presente sin pasado y, por ello, la rehabilitación integrada pretende construir el patrimonio del futuro sobre el patrimonio heredado.
\end{abstract}

\section{RÉSUMÉ}

La faillite de l'urbanisme positiviste des ans soixante y soixante-dix précipite á la décade des quatre vingts de nouveaux concepts et de pratiques d'intervention à la ville. L'urbanisme «requalificateur» de la dernière génération à nos Centres Historiques se constitue en alternative a l'ultraconservatisme et à la rénovation spéculative, en mettant en evidence que la ville n'est pas un moyen facile et rapide de gagner beaucoup d'argent, mais elle est le cadre de vie quotidienne en commun soumise à des régles claires de jeu. Il n'existe pas de présant sans passé et, c'est pourquoi, la réhabilitation integrée essaye de construire le patriomoine de l'avenir sur le patrimoine hérité.

* Los artículos de los Dres. Campesino Fernández y Gozálvez Pérez, corresponden a sendas conferencias dictadas en la Reunión Científica sobre «La utilización de los estudios geográficos en la planificación del territorio», organizada por el Departamento de Geografía Humana, Instituto Universitario de Geografía de la Universidad de Alicante, con el patrocinio de la Dirección General de Investigación Científica y Técnica; Conselleria de Cultura, Educació i Ciencia de la Generalitat Valenciana, y Caja de Ahorros provincial de Alicante. 


\section{El urbanismo español de la última generación}

El urbanismo es una disciplina que permite al geógrafo no sólo conocer de forma aséptica el funcionamiento de la ciudad, sino intervenir en ella a través del planeamiento, porque hacer ciudad es un pacto arbitrado entre dos distintos grupos sociales de una comunidad mediante un documento concreto y legible, que debe someter los intereses y las estrategias contrapuestas de los distintos agentes urbanos a unas normas claras de juego en beneficio de la colectividad. Ello será posible siempre que los responsables de la gestión tengan claros sus objetivos de política urbana, cumplan y hagan cumplir el marco jurídico vigente. No es así, porque nuestros supuestos representantes políticos no dan la talla y los profesionales cuestionarnos también la operatividad de la actual legislación urbanística.

La revisada Ley 19/1975 de Régimen del Suelo y Ordenación Urbana y su normativa reglamentaria (B.O.E. 1979) ${ }^{1}$ se redactaron en el marco urbano expansionista de 1972, tornado recesivo a la hora de su aplicación a finales de los setenta en una coyuntura de incertidumbre política y económica, ajena a su vez a la realidad presente que reclama una necesaria readaptación.

Las nuevas disposiciones urbanísticas se solapan con las atribuciones que el Texto Constitucional otorga a las Comunidades Autónomas y a los Ayuntamientos democráticos, generando un caos de competencias entre las diversas instancias administrativas (estatal, autonómica y local). A partir de las transferencias de ordenación urbana y territorial se tiende al canibalismo por apropiación de las del nivel inmediato inferior. El traspaso de competencias ha impedido afrontar la modernización de la legislación urbanística, produciéndose una disociación efectiva entre el marco jurídico y la práctica del planeamiento a diferentes escalas.

Algunas Comunidades Autónomas (Cataluña, Madrid, Galicia) han puesto en marcha programas para control de las urbanizaciones ilegales e implantación de la disciplina urbanística en el suelo no urbanizable, pero la clave del desbarajuste normativo y de la falta de ideas se encuentra en la relación entre planeamiento y ordenación del territorio, porque ésta no puede concebirse como un sumatorio de políticas urbanísticas municipales, ni entenderse que mientras desaparecen las áreas metropolitanas de Madrid, Bilbao, Valencia y Barcelona, la reciente Ley de Régimen Local permita que se promuevan otras, como la de Sevilla.

A la falta de ideas y de capacidad financiera los gobiernos locales unen la impotencia técnica para asumir las nuevas competencias urbanísticas, por lo que tan sólo media docena de ciudades pueden desarrollar planeamiento general controlado.

No debe extrañar en un país con tal diversidad de núcleos urbanos y de situaciones solapadas en la última década, desde la regresión y el bloqueo demográfico al crecimiento desordenado de las ciudades turísticas litorales, que pone en tela de juicio el concepto de crisis urbana generalizada. Crisis concebida como regresión ambiental y social tanto de las periferias como de los barrios centrales, con mayor afección en el caso de ciudades de tamaño superior insertas en regiones intensamente industrializadas (FERRER, 1987) ${ }^{2}$.

El bloqueo de la ciudad ilimitada es el colofón lógico de la práctica de los modelos urbanos funcionalistas. Factores como la estabilización del crecimiento demográfico, el

1 BOLETÍN OFICIAL DEL ESTADO: (1979). Régimen del Suelo y Ordenación Urbana. Textos legales, 43. Madrid, Gabinete Jurídico-Administrativo.

2 FERRER, M.: (1987). «La naturaleza de la crisis urbana. El caso vasco». II Congreso Mundial Vasco, Congreso de Geografía. Ponencia. Vitoria-Gasteiz, Gobierno Vasco. 27 págs. mecanografiadas. 
colapso del subsector de la construcción, la escalada inflacionista, el aumento de la presión fiscal, la crisis de comercialización del parque inmobiliario construido, la ausencia de demanda solvente y la dificultad de acceso de la mayoría de la población a los créditos hipotecarios constituyen razonamientos sólidos para repensar la ciudad.

La quiebra profunda del urbanismo positivista de los años 60 y 70 (TERAN, 1984) ${ }^{3}$ precipita en los ochenta un nuevo concepto de intervención en la ciudad. El cambio de orientación del urbanismo supondrá modificaciones conceptuales, de escala y de metodología analítica.

Desde la búsqueda de la calidad formal del urbanismo diseñado hasta el equilibrio compensatorio de los déficits de servicios y equipamientos comunitarios, pasando por la conservación y reutilización del centro-ciudad, el «nuevo urbanismo» regeneracionista y rehabilitador se constituye en ruptura alternativa frente a la renovación salvaje, tratando de evidenciar que la ciudad no es un instrumento de hacer dinero fácil y rápido, sino el marco de convivencia diaria.

Potenciar el urbanismo como función pública frente a la inercia de la especulación inmobiliaria y a la impunidad de los particulares genera hoy las máximas tensiones en el suelo urbano consolidado, que se debate entre la rehabilitación y la obra nueva, en el mejor de los casos entendida como patrimonio arquitectónico de futuro.

El problema es grave porque al ser el espacio de mayor valor añadido permite repercutir todo tipo de cargas y beneficios sobre ventas y alquileres, resistiéndose por ello los dueños del suelo al hecho de que la regulación del contenido del derecho de propiedad es competencia autonómica, plasmada en las diferentes calificaciones del planeamiento urbanístico como preceptúa la legislación.

Asistimos a un repliegue del fracasado planeamiento de escalas territoriales superiores hacia los problemas patrimoniales y medioambientales del espacio urbano concreto. Las disfuncionalidades y contradicciones internas del modelo urbano racionalista invierten hoy el proceso iniciado en la década de los sesenta. La búsqueda de las señas de identidad perdidas en la marginalidad de las periferias urbanas convierte a los centros históricos en el objetivo de un planeamiento posibilista, negociable, comprometido con la realidad y supuestamente democrático (CAMPESINO, 1986) ${ }^{4}$, porque la participación continúa en un nivel de utopía.

El tecnocratismo elitista de laboratorio, de influencia anglosajona, deja pasar a una investigación comprometida de base histórica y marcado contenido social que exige un conocimiento integrado de los problemas de los centros históricos para la superación de sus limitaciones, a partir de propuestas alternativas de revitalización global y evidente sabor mediterráneo.

En el momento presente no hay modelos válidos, porque cada ciudad debe ser modelo de sí misma (QUERO, 1981) , lo que exige un análisis propositivo con estrecha relación proyecto-lugar, para descubrir lo específico de cada barrio y de cada situación urbana frente a la «lógica» de la ciudad.

3 TERÁN TROYANO, F. de: (1984). «Teoría e intervención en la ciudad, balance de un período. Estado de la cuestión. Perspectivas». Ciudad y Territorio. 59/60. Madrid. IEAL.

4 CAMPESINO FERNÁNDEZ, A. J.: (1986). «Revalorización funcional de los centros históricos españoles». Estudios sobre Espacios Urbanos, pp. 91-103. Madrid, IEAL y Casa de Velázquez. Véanse asimismo, (1984). «Los centros históricos: análisis de su problemática». Norba, Revista de Geografia, V, 51-61. Cáceres, Universidad, y (1987). «Patrimonio y planeamiento democrático en nuestras ciudades históricas». IV Coloquio Ibérico de Geografia, pp. 545-554. Coimbra, Instituto de Estudios Geográficos.

5 QUERO CASTANYS, D.: (1981). «Nuevos enfoques para el planeamiento General de Ordenación Urbana. Apuntes para una ponencia». CEUMT, 35, 19-22. Barcelona. 
Este nuevo planteamiento impone la rigurosa aplicación de una metodología historicista interdisciplinar, que hace primar la intuición sobre la racionalidad y que no ha de limitar la capacidad creadora, porque para Fernando de TERAN se debe «conservar lo que valga la pena y perder el miedo a hacer buenas obras con el lenguaje de hoy» (AA.VV. 1987) ${ }^{6}$, si queremos crear el patrimonio del siglo XXI.

El urbanismo español de la última generación se mueve bajo la influencia de la teoría de la recuperación de la ciudad, de gran atractivo en todo el mundo por su procedencia italiana (CIARDINI/FALINI, 1983) ${ }^{7}$. Desde la arquitectura, Luis RODRÍGUEZ AVIAL constata el hecho de que «hace veinte años nadie defendía en las Escuelas el cariño por la ciudad en la que uno vivía y por el patrimonio edificado. Los locos de entonces son comprendidos hoy en esta faceta, incluyendo a algunos promotores y propietarios»; ahora un buen número de profesionales defienden el proyecto urbano y el diseño como métodos de intervención para control de la forma urbana.

Se está abordando la gestión parcial de la ciudad y el debate se centra entre rehabilitar o hacer de nuevo, porque al mantenerse bloqueado el planeamiento superior hay dudas sobre la operatividad final de las intervenciones fragmentadas, ya que por sectoriales, al no estar reguladas por un documento de rango superior, propuestas y ordenanzas desagregadas colisionan y se tornan contradictorias. Debe existir un proyecto unitario que permita coordinar los distintos niveles y estrategias.

Y ello debido a que el centro-ciudad es el ámbito territorial más intensamente humanizado que puede estudiar un geógrafo, un laboratorio al aire libre donde la trama de las interacciones alcanzan las más altas cotas de complejidad, y cuya patología demandan intervenciones integradas, porque la salvaguardia desde la cultura de protección se revela insuficiente para afrontar el deterioro físico, social, económico y ambiental de nuestros centros históricos.

\section{Rehabilitación como alternativa}

La estrategia de renovación con bulldozer en los centros históricos despierta durante el último lustro las iras de los estratos más cultos de las sociedades europeas ante la devastación del patrimonio heredado.

El lema de «ciudades para vivir» sirve de estandarte a la Campaña Europea del Renacimiento de la Ciudad del Consejo de Europa, 1980-1981, que propone el mecanismo de rehabilitación integrada, como el conjunto de actuaciones coherentes y programadas tendentes a potenciar los valores socioeconómicos, ambientales, edificatorios $\mathrm{y}$ funcionales de determinadas áreas urbanas y rurales, con la finalidad de elevar la calidad de vida de la población residente, mediante alternativas para la mejora de las condiciones del soporte físico, la elevación de los niveles de habitabilidad y uso, la dotación de equipamientos comunitarios, servicios y espacios libres de disfrute público.

A partir de este momento, la Dirección General de Arquitectura y Vivienda considera la rehabilitación integrada como un instrumento de reequilibrio territorial urbano para la comprensión global de las interacciones formales, sociales y funcionales que afectan al

6 AA. VV. (1987). «El urbanismo español de la última década. Mesa Redonda». Urbanismo. Revista del Colegio Oficial de Arquitectos de Madrid, 1, 9-24. Madrid. Servicio de Publicaciones del COAM.

7 CIARDINI, F. FALINI, P.: (1983). Los centros históricos. Política urbanística y programas de actuación. Barcelona, Gustavo Gili. (Edición original de 1978). 
espacio interno de la ciudad (GARCÍA FERNÁNDEZ, y OTROS, 1982) ${ }^{8}$. Objetivos que van a ser prioritarios en la política urbana actual por tratarse de una línea de actuación socialmente avanzada, económicamente racional y de gran coherencia urbanística, mediante la articulación de un soporte legal específico (DECRETOS, 1980-1987)9 .

Medio siglo ha transcurrido desde la pionera Ley de 13 de mayo de 1933, del Tesoro Histórico-Artístico. La reciente Ley 13/1985, de 23 de junio, del Patrimonio Histórico Español (B.O.E. 1985) ${ }^{10}$ y el Real Decreto $111 / 1986$, de 10 de enero que la desarrolla, consagran un nuevo concepto de patrimonio histórico, como herencia cultural cuyo conocimiento, respeto, acrecentamiento, disfrute y transmisión a las generaciones futuras permitirán el hallazgo de las señas propias de identidad y la conciencia de arraigo a la tierra. De esta forma la rehabilitación del patrimonio es concebida como el objetivo máximo de la planificación y de la gestión urbanas.

No puede decirse que hasta el momento haya elementos suficientes de referencia para una valoración de lo realizado, sobre todo en lo referente a rehabilitación de viviendas e introducción de la política de alojamientos de protección oficial en los cascos antiguos. En esta materia nos encontramos en mantillas, por la disociación entre la política estatal del (IPPV) Instituto para la Promoción Pública de la Vivienda y las competencias municipales para la incorporación de VPO a los centros históricos, la juventud de la legislación rehabilitadora, que ha llegado tarde, el raquitismo financiero, la complejidad de los procesos, el desinterés inicial de los técnicos y la manifiesta incapacidad de las Corporaciones para crear patrimonios municipales de suelo con los que orientar una política de vivienda más justa, contrapesando así la elevación artificial del precio del suelo y la rentabilidad expectante.

Hasta la aparición del R. D. 2329/83, el desinterés de los agentes urbanos y la inercia conservacionista hacen de la rehabilitación una práctica indiferenciada y puntual en las que prima la protección física de edificios singulares con valores arquitectónicos sobre la política integrada de atención a consideraciones sociales y funcionales (ARRIBAS, 1982) ${ }^{11}$.

Como si de la recuperación de un miembro corporal se tratase, la rehabilitación de los centros históricos no puede limitarse a un maquillaje de restitución de sus aspectos externos, sino que debe garantizar la revitalización de los valores de uso y funcionamiento, para un correcto dinamismo del conjunto urbano. De lo contrario, la rehabilitación se queda en cirugía estética.

La experiencia europea en materia de rehabilitación del patrimonio demuestra que el valor esencial de los centros históricos reside en la capacidad de adaptación sucesiva a las mutaciones de la ciudad en su conjunto, y que las pretensiones de convertir el centro-ciudad en museo es sacrificarlo, porque tanto la renovación salvaje como la conservación a ultranza

8 GARCÍA FERNÁNDEZ, E. y otros: (1982). Rehabilitación integrada de Cudillero. Madrid, MOPU.

9 Consúltense al respecto: Real-Decreto-Ley 12/1980, de 26 de septiembre, sobre actuaciones del estado en materias de vivienda y suelo. Real Decreto 375/1982, de 12 de febrero, que desarrolla el anterior. Real Decreto 2555/1982, de 24 de septiembre sobre Áreas de Rehabilitación integrada, y la Orden de 24 de noviembre de 1982 que lo desarrolla. Real Decreto 2329/1983, para delimitar con mayor precisión el marco legal de las A.R.I. Real Decreto 1985 de 6 de noviembre, sobre municipios de rehabilitación preferente, y Real Decreto 223/1987, de 20 de febrero en materias de préstamos bancarios.

10 BOLETÍN OFICIAL DEL ESTADO: (1985). «Ley del Patrimonio Histórico Español», núm. 155, de 29 de junio. Madrid.

11 ARRIBAS, P.: (1982). «El urbanismo en la ordenación y protección de los valores del patrimonio histórico-artístico». Estudios Territoriales, 8, 121-132. Madrid, MOPU. 
provocan resultados negativos. Por ello, en los programas de rehabilitación de la ciudad histórica $(\mathrm{POL}, 1983)^{12}$ hay que tender tanto a la protección de los elementos y ambientes, cuanto a su readaptación global a las cambiantes necesidades urbanas.

Estas carencias motivan en 1983, un año después de nuestro ingreso en el grupo de países firmantes del Patrimonio Cultural de la Humanidad, la primera reunión en Toledo de alcaldes de cinco ciudades españolas -Cáceres, Granada, Salamanca, Santiago y Toledo-, singulares por sus contenidos históricos y por los graves problemas de deterioro físico, social y funcional, para recabar ayuda financiera a la rehabilitación. Las esperanzas de respuesta parecían reforzarse al incluir la UNESCO en el P.C.H. a las ciudades de Ávila y Segovia, el 4 de diciembre de 1985, y a Toledo y Cáceres, el 25 de noviembre de 1986. La falta de respuesta positiva ha forzado la última reunión en Évora, 18 de septiembre de 1987, y de nuevo en Toledo el 5 de octubre, para insistir en las reivindicaciones de ayuda financiera, que en este último caso se cifra en 2.000 millones de pesetas.

Por parte de los Ayuntamientos existe una legítima aspiración a canalizar mediante el planeamiento todas las actuaciones en conjuntos histórico-artísticos, reivindicando la dispersión de competencias que se camuflan entre la Administración Central y las Comunidades Autónomas. La ordenación del suelo consolidado viene a constituir la gran preocupación del urbanismo de finales de los ochenta. El nuevo tratamiento que el legislador otorga a la regulación del suelo urbano aparece ahora como un reto que hay que afrontar: rehabilitar la estructura urbana, apoyándose para ello en una normativa suficiente de planificación.

Los Planes Especiales de Protección (P.E.P.R.I.) se insertan dentro de la tipología de figuras legales para el planeamiento Urbanístico del Territorio (arts. 17 a 25 de la Ley del Suelo y 76 a 85 del Reglamento de Planeamiento). Se han consolidado como documento básico del nuevo planeamiento en Áreas de Rehabilitación Integrada (A.R.I.) y Conjuntos Histórico-Artísticos (C.H.A.), caracterizándose por la ordenación de los mismos desde una perspectiva sectorial flexible, adecuada a la problemática especial que aconseja su formulación (MARTÍNEZ, y OTROS, 1985) ${ }^{13}$.

\section{La aportación de los geógrafos profesionales}

El reto urbanístico de finales de los ochenta obliga al geógrafo urbano a descender en las escalas de análisis e introducirse de lleno en el corazón de la ciudad, por definición arrítmico, anémico, depauperado y con graves problemas de arteriosclerosis.

El centro-ciudad exige un tratamiento integral, aún desconocido, porque el planeamiento que se redacta en ocho meses es incapaz de ahondar en la comprensión de un pasado urbano tan complejo y de un presente tan crítico, careciendo, por lo general, de rigor y limitándose a dar cumplimiento a las bases de la convocatoria.

Por esta razón, tratamos de aportar un diseño metodológico preciso y fundamentado por nuestras fuentes geográficas de análisis urbano para la redacción del planeamiento de rehabilitación integrada, y ofrecerlo con criterios abiertos e interdisciplinares a las Corpora-

12 POL, F.: (1983). «De la política de protección a la estrategia de rehabilitación urbana: iniciativas municipales y políticas estatales». Madrid: de la restauración singular a la rehabilitación integrada, pp. 68-83. Madrid, MOPU.

13 MARTÍNEZ, C. y otros: (1985). La ordenación urbana. Aspectos legales y práctica profesional. Pamplona, EUNSA. 
ciones, responsables de la redacción de los pliegos de condiciones técnicas y administrativas y de la selección de los equipos redactores de los P.E.P.R.I., anticipándonos a la profusión de documentos que presumiblemente van a salir a concurso en esta loca carrera que tiene como destino la tópica fecha de 1992, especialmente en Extremadura, la «tierra donde nacieron los dioses...» del descubrimiento.

La propuesta metodológica del GRÁFICO I responde al organigrama de contenidos legales y plan de etapas de un P.E.P.R.I. y está concebida con una concepción sistémica e integradora. Un documento de planificación de estas características debe clarificar:

a) Justificación de fines y objetivos adecuados a las necesidades del marco espacial al que se destina. Nosotros vamos más lejos cuando proponemos que en esta fase introductoras sea el equipo redactor el que defina ante el Ayuntamiento, con razones científicas, el perímetro de actuación en el centro histórico, y no que sus servicios técnicos lo impongan arbitrariamente en la convocatoria del concurso. A nadie se le oculta, y menos a los geógrafos, la pluralidad de aureolas de «protección» otorgadas a los Conjuntos Histórico-Artísticos, ni el deliberado confusionismo y la variedad de interpretaciones acerca de los límites del centro histórico, sujetas al libre arbitrio de los encontrados intereses de los agentes urbanos, desde la propia Corporación a los Colegios profesionales, Comisiones Provinciales de Urbanismo, promotores inmobiliarios y detentadores de la propiedad.

Un planeamiento especial que no parta de una clarificación razonada del perímetro de actuación, consensuada con el organismo contratante, afronta el riesgo de no gestionarse, al convertirse sus bordes de contacto en terreno abonado para las alegaciones. Definir en origen esa frontera concreta sin arbitrismo exige un conocimiento previo de la dinámica urbana, que difícilmente puede lograrse sin la presencia y añosa aportación investigadora de los geógrafos al equipo redactor.

b) Memoria y Estudios Complementarios constituyen el documento base de Información, que, partiendo de un prediagnóstico urbano y de la elección de troncos temáticos de investigación (1. ${ }^{\mathbf{a}}$ fase), analiza los aspectos básicos de la situación de partida del área, evalúa el comportamiento de las variables y justifica la finalidad del planea lento por referencia a los problemas diagnosticados y a los objetivos públicos expresos (2. ${ }^{a}$ fase), cataloga el patrimonio

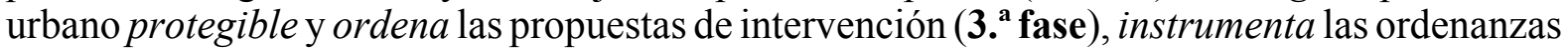
y el estudio económico-financiero ( $4 .^{a}$ fase $)$, para que la Corporación tramite el documento final, sometiéndolo a información pública, alegaciones, aprobación y gestión.

La Memoria ha dejado de ser el ladrillo erudito de trámite para convertirse en la filosofía del planeamiento, porque de la calidad del análisis, morfoestructural, social y funcional, y del diagnóstico integrado de los problemas dependerán las intervenciones en el centro histórico.

c) Planimetría de información y ordenación a escalas adecuadas se configura como indispensable soporte cartográfico y fotogramétrico de la Memoria, entre 1:500 y 1:2.000, para el grafiado de problemas y soluciones urbanísticas: determinaciones del Plan General en los subsectores afectados, topografía, catastro parcelario, alturas, estado de la edificación, estructura urbanas, propiedad del suelo, zonificación de usos, servicios generales, equipamientos comunitarios, red viaria, tráfico y unidades de interés cultural, paisajístico o medioambiental. Las minutas de los vuelos específicos se convierten en material insustituible del planeamiento para el análisis del entramado, textura y granulado de áreas urbanas residenciales, tipología de usos del suelo, afecciones topográficas, accesibilidad y entornos ambientales en el análisis micromorfológico del centro-ciudad.

Nuestra aportación en el rastreo de la cartografía histórica, búsqueda de planos y proyectos de edificación antigua es indiscutible al tratarse de una dura labor de archivo que nadie piensa disputarnos. La cartografía urbana es un arma de control político del territorio 


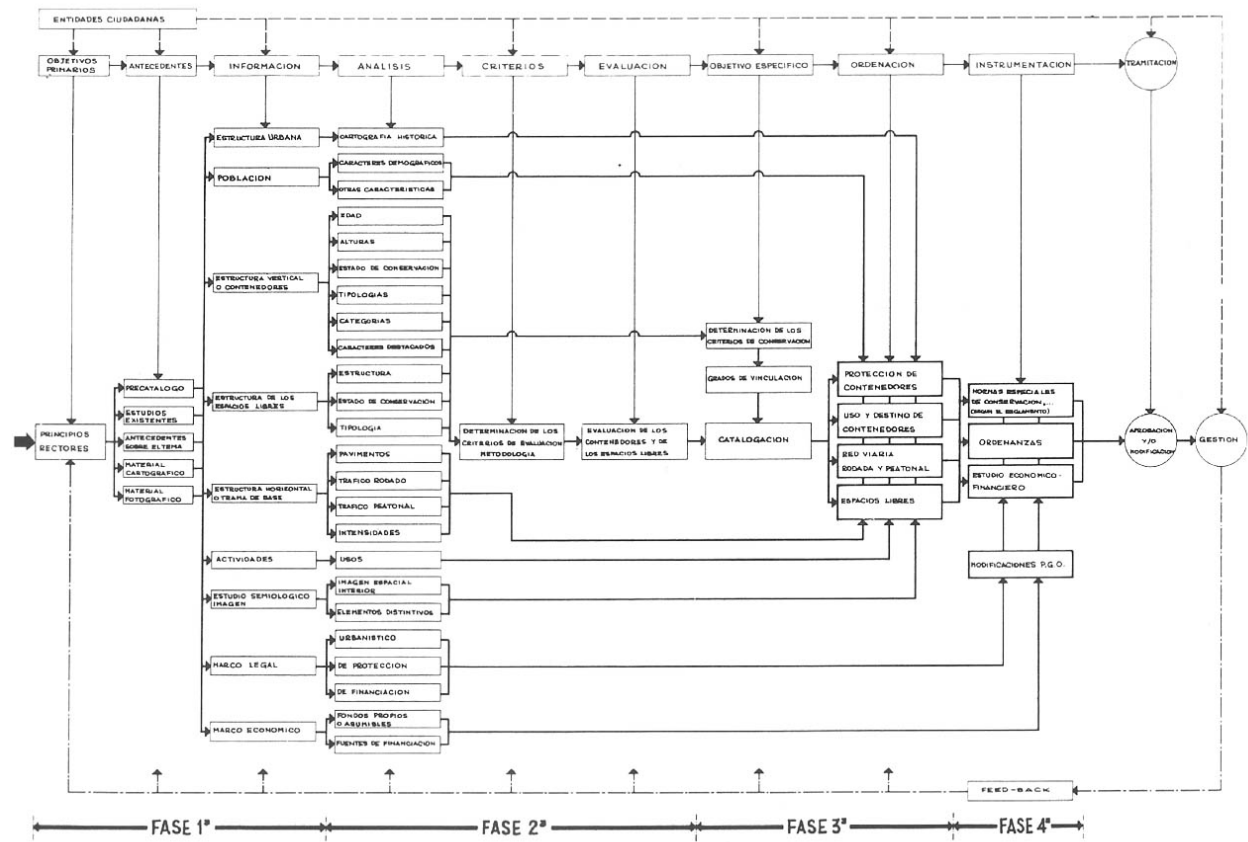

GRÁFICO I. Centros históricos de Extremadura. Planes especiales de rehabilitación. Propuesta metodológica. Plan de trabajo. 
en manos de administradores públicos irresponsables y de especulación en manos de particulares, mientras que para el geógrafo es su herramienta de trabajo, de diálogo de fructífera discusión científica con los restantes profesionales, Administración y particulares para la plasmación de propuestas legibles que permitan la ordenación de la ciudad. Es justo reconocer que la interdisciplinariedad del planeamiento enriquece nuestra disciplina.

d) Normativa de protección que recoja los criterios básicos y grados de vinculación para confeccionar las ordenanzas urbanísticas, del P.E.P.R.I., en consonancia con las establecidas por el P.G.O.U. y las municipales vigentes de edificación: calificación de suelo y usos pormenorizados, estudios de detalle, proyectos de urbanización, condiciones higiénicas de las viviendas, tratamiento, uso y conservación de los edificios protegidos y espacios libres, condiciones estéticas y ordenanzas específicas por manzanas.

Si bien debemos ceder en este apartado el lugar que le corresponde a los expertos en derecho urbanístico, el rastreo de Reglamentos y Ordenanzas Municipales históricas se convierte en tarea del geógrafo, por cuanto su investigación urbana se fundamenta también en este tipo de fuentes.

e) Estudio económico-financiero para ajuste de las soluciones propuestas en el correspondiente Plan de Etapas y de los mecanismos urbanizadores (expropiación, compensación, permuta, reparcelación y reequipamientos) en cada una de las Unidades de Actuación, según el ritmo de las inversiones públicas y privadas.

\section{A modo de propuestas}

- La nueva política urbanística debe ordenar y mejorar el espacio interno de la ciudad y poner punto final a la desintegración de los centros históricos, impidiendo que desaparezcan la simbología y las señas de identidad.

-El espacio político del urbanismo debe configurarse desde las decisiones ciudadanas, porque los políticos responsables han sido elegidos y los técnicos lo son por mandato burocrático, por lo que el urbanismo debe dejar de ser un saber esotérico y complicado en manos de unos pocos.

- La ciudad es de todos porque está constituida con la yuxtaposición y sobreimposición de contenidos culturales de generaciones pretéritas, por lo que no es aconsejable identificar el interés general con la suma de los intereses particulares de los agentes urbanos de siempre, pero con distintas estrategias.

- La rehabilitación es hoy una práctica innovadora en términos técnicos y culturales, frente a la renovación salvaje del urbanismo de urgencia y despilfarro.

- Representa una revolución conceptual y exige una metodología revolucionaria en el sentido de que apostar por el futuro en estos términos significa dar un sentido racional a la ordenación del territorio, para no ver anulada nuestra historia y nuestra maravillosa cultura mediterránea, en lo que parece existir demasiado interés.

- La política de rehabilitación se configura como un mecanismo de intervención multiforme por el abanico de casos individuales que no pueden ser tratados en serie: numerosos pequeños propietarios, los más frágiles y menos cualificados del espectro urbano que ocupan un parque inmobiliario antiguo y degradado, cuyo derecho a una vivienda digna choca con la complejidad de funciones concentradas en el centro-ciudad.

-Política que requiere operaciones gigantescas en las que es preciso realizar toda una gimnasia de coordinación para integrar las estrategias de los distintos agentes en un proyecto municipal coherente en su programación y gestión.

-En este arbitrio de intereses, que en el fondo han de solucionarse para el bien general 
de la ciudad, es donde se le exige a la iniciativa pública estar a la altura de las circunstancias, como árbitro y motor de la iniciativa privada, pues de lo contrario el alza espectacular del suelo central puede desembocar, como ya está aconteciendo en el caso de Madrid, en que la rehabilitación sea el gran negocio especulativo de finales de siglo.

- Las herramientas disponibles para hacer frente a una política de rehabilitación integrada mediante planeamiento especial permiten a los Ayuntamientos:

a) resolver los problemas urbanísticos delimitando las áreas de actuación, etapas y medios técnico-financieros de gestión;

b) invertir en la adquisición de edificios y de suelo para incrementar el patrimonio municipal, convirtiéndose en empresa para la promoción y adjudicación de viviendas en propiedad o alquiler a los residentes de mayor precariedad económica, procurando preveer el problema de los realojos durante el período de rehabilitación, favoreciendo la participación de los usuarios en la gestión y regulando los deberes que contraen en evitación de especulaciones encubiertas,

c) incitar y obligar en su caso a la propiedad privada a la realización de los oportunos proyectos de mejoras y mantenimiento del parque inmobiliario, adquiriendo, permutando, compensando o expropiando viviendas y solares cuyos propietarios se desentiendan reiteradamente de su conservación.

No hay presente sin pasado, y por ello la rehabilitación integrada representa la compleja alternativa urbanística de creación del patrimonio futuro sobre el patrimonio heredado.

\section{REFERENCIAS BIBLIOGRÁFICAS}

AA. VV.: (1985). El territorio de los años 80. Concepto, problemas y propuestas. Madrid, MOPU-DGATU.

AA. VV.: (1986). Introducción al diseño urbano. La calidad de la ciudad consolidada. Madrid, MOPU-ITUR. AA. VV.: (1986). Planeamiento especial y rehabilitación urbana. Madrid, MOPU-FEMP.

ADRIÁN, J.: (1987). «El Plan Especial del Casco Medieval de Vitoria». Urbanismo, Revista del Colegio Oficial de Arquitectos de Madrid, 2, 62-66. Madrid, COAM.

AYUNTAMIENTO DE MADRID: (1986). Plan Especial Villa de Madrid: 14 años de gestión. Madrid, Gerencia Municipal de Urbanismo.

AZCARATE, B. y CAMPESINO, A. J.: (1987). «Política de rehabilitación en los centros históricos de Europa: la República Federal de Alemania». X Congreso Nacional de Geografía, pp. 493-502. Zaragoza, AGE y Departamento.

BOHIGAS, O.: (1986). Reconstrucción de Barcelona. Madrid, MOPU-Arquitectura.

BUSQUETS, J. y otros: (1985). Evaluación de las necesidades de rehabilitación. Madrid, MOPU-DGATU.

CAMPOS, M. ${ }^{\mathrm{a}}$ L.: (1987). «La política de rehabilitación y sus actuaciones en el casco histórico de Toledo: la rehabilitación residencial». X Congreso Nacional de Geografía, pp. 503-516. Zaragoza, AGE y Departamento.

FEDERACIÓN INTERNACIONAL DE VIVIENDA Y URBANISMO: (1987). Una nueva política de vivienda y urbanismo para las ciudades históricas. Congreso Internacional. Sevilla, FIVU. GARCÍA FERNÁNDEZ, J. L. e IGLESIAS, L. S.: (1986). La Plaza en la ciudad. (Galicia, Asturias, Cantabria, País Vasco, Navarra). Madrid, Herman Blume.

ITUR-MOPU-AYUNTAMIENTO DE SALAMANCA: (1987). Salamanca. Plan Especial de Protección y Reforma Interior del Recinto Universitario y Zona Histórico-Artística. Madrid, ITUR-MOPU y A. S. 
KLEYMANS, J. (1987). «Da renovação urbana a reabilitação das zonas antigas das cidades en França y em Portugal». IV Coloquio Ibérico de Geografía, pp. 555-560. Coimbra, Instituto de Estudios Geográficos. MARBACH, A. (1986). «L'expérience française de conservation et mise en valeur des centres historiques». Estudios sobre Espacios Urbanos, pp. 791-802. Madrid, IEAL y Casa de Velázquez.

MERLIN, J.: (1986). «Rehabiliter et revitaliser les vieux centres historiques». Estudios sobre Espacios Urbanos, pp. 821-832. Madrid, IEAL y Casa de Velázquez.

PATRONAT MUNICIPAL DE L'HABITATGE: (1985). «La Rehabilitació a Barcelona». Habitatge, 4. Monográfico. Barcelona, P.M.H.

PERCOURT, J.: (1986). «El diseño urbano como filosofía del planeamiento». Ciudad y Territorio, 67, 35-43. Madrid, IEAL.

RAMOS, A. y QUIÑORERO, F.: (1987). «Degradación y planeamiento en el centro histórico de la ciudad de Alicante». X Congreso Nacional de Geografia, pp. 603-613. Zaragoza, AGE y Departamento.

VALENZUELA, M.: (1987). «Ciudad y calidad de vida, Políticas e instrumentos para la recuperación social del espacio urbano». II Congreso Mundial Vasco. Congreso de Geografía, Ponencias, pp. 79-108. Vitoria-Gasteiz, Gobierno Vasco. 